\title{
Prediction of the surface structures resulting from heat accumulation during processing with picosecond laser pulses at the average power of $420 \mathrm{~W}$
}

\author{
Sebastian Faas ${ }^{1}$ (D) Uwe Bielke $^{1} \cdot$ Rudolf Weber $^{1} \cdot$ Thomas Graf $^{1}$
}

Received: 21 March 2018 / Accepted: 11 August 2018 / Published online: 14 August 2018

(c) The Author(s) 2018

\begin{abstract}
Heat accumulation due to successive laser pulse (HAP) incident on the same spot and heat accumulation due to successive scans (HAS) of the laser beam over the same spot significantly influences the process result, especially when the resulting temperature of the workpiece exceeds the melting temperature. In particular, heat accumulation is one of the dominating effects during short-pulse laser functionalization of surfaces and strongly affects the resulting surface structures. Within this study, a novel heat accumulation model is introduced to calculate the temperature increase in the workpiece for the whole process including the effects of HAP and HAS in which the latter is differentiated between heat accumulation due to multiple passes (HAS-I) and heat accumulation due to multiple layers (HAS-II). With the new model, the surface structure was successfully predicted when using an ultra-short pulsed (USP) laser with an average power of $420 \mathrm{~W}$ for laser surface structuring of polished AISI 316L.
\end{abstract}

\section{Introduction}

Heat accumulation occurs when the time between successive heat inputs on the same spot is too short for the processed material to cool down to the initial temperature [1]. The thus occurring gradual temperature increase eventually causes the temperature of the workpiece to exceed a given critical value $T_{\text {crit }}$, e.g., the melting temperature $T_{\text {melt }}$. Heat accumulation can, therefore, constitute a distinct processing limit $[2,3]$. When processing with a pulsed laser beam, the heat accumulation from pulse to pulse (HAP) is determined by the number $N_{\text {pps }}=d_{\mathrm{s}} \cdot f / v_{\text {feed }}$ of pulses that are incident at one spot, where $d_{\mathrm{s}}$ is the diameter of the laser beam on the surface of the workpiece, $f$ is the pulse repetition rate of the laser, and $v_{\text {feed }}$ is the feed rate.

In many processes, e.g., surface structuring and surface ablation, the workpiece is processed along parallel arranged lines [4-7] as exemplarily sketched in Fig. 1a. The solid red arrows represent the path of the center of the laser beam during the processing of the surface and the dashed red

Sebastian Faas

sebastian.faas@ifsw.uni-stuttgart.de

1 Institut für Strahlwerkzeuge (IFSW), University of Stuttgart, Pfaffenwaldring 43, 70569 Stuttgart, Germany lines indicate the positioning of the laser beam between two successive processing lines. During this repositioning step, the laser is off. The length of the processing lines is $\ell$ and the distance between the adjacent lines is $d_{\ell}$. An alternative scanning strategy with shorter repositioning paths and alternating feed direction on the processing lines is discussed in the "Appendix". When the hatching distance $d_{\ell}$ of adjacent lines is smaller than the focal diameter $d_{\mathrm{s}}$, every spot within the processed area is scanned more than once. This is shown in Fig. 1b, with an example showing one pulse for each processing line to visualize the multiple interactions with a given point as marked by the white cross. This results in a number $N_{\text {passes }}=d_{\mathrm{s}} / d_{\ell}$ of passes of the laser beam over one spot which causes the heat accumulation between multiple scans (HAS-I). The time interval between two successive passes depends on the scanning strategy.

Figure 1 illustrates the path of the laser beam covering a given area to ablate one layer of the material. The ablation of several layers is usually necessary to reach the desired ablation depth. The number $K_{\text {layers }}$ of layers required to reach the desired depth depends on the amount of ablation obtained by one single-processing layer which on its turn depends on the processing parameters. Different orientations of the beam trajectories for subsequent layers may be applied. The simplest method is to proceed the same way on every processing layer. Better processing results may be achieved when 


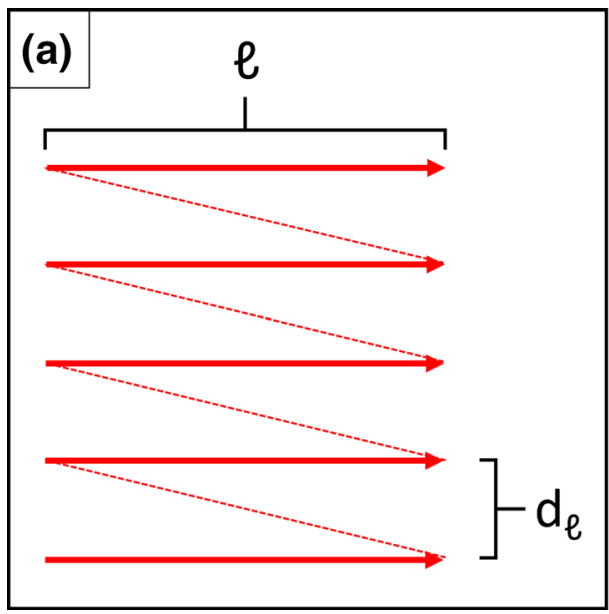

Fig. 1 a Schematic illustration of a typical scanning strategy. The solid red arrows show the movement of the laser beam during processing, the dashed lines indicate the repositioning of the beam (during which the laser is off). $\ell$ is the length of the processing paths and $d_{\ell}$ the distance between adjacent paths. All processing lines start on

rotating the beam trajectory by an angle, e.g., $45^{\circ}$ or $90^{\circ}$ for each subsequent processing layer as, e.g., reported in [7]. Additionally, the feed rate or the hatching distance may also be changed. The processing strategy, therefore, determines the time elapsing from layer to layer.

For the present study, a home-build mJ-ps-laser (wavelength $\lambda=1030 \mathrm{~nm}$, pulse duration $8 \mathrm{ps}$, pulse energy $E_{\mathrm{P}}=1.4 \mathrm{~mJ}$, pulse repetition rate $f=300 \mathrm{kHz}$ ) was used [8]. Between two successive pulses, the thermal diffusion length is $\ell_{\text {diff }}=(4 \cdot \kappa / f)^{0.5}=7.11 \mu \mathrm{m} \mathrm{[9],} \mathrm{where} \kappa=3.75 \times 10^{-6} \mathrm{~m}^{2} / \mathrm{s}$ is the thermal diffusivity of the processed material. Since this is small compared to the diameter $d_{\mathrm{s}}=500 \mu \mathrm{m}$ of the beam on the workpiece used in this study, 1D heat flow can be assumed for the time between two consecutive pulses [9]. For the larger number $N_{\mathrm{pps}}=d_{\mathrm{s}} \cdot f / v_{\text {feed }}$ of pulses incident on one spot during a single pass of the laser beam, the thermal diffusion length modifies to $\ell_{\text {diff }}=\left(4 \cdot \kappa \cdot N_{\mathrm{pps}} / f\right)^{0.5}=($ $\left.4 \cdot \kappa \cdot d_{\mathrm{s}} / v_{\text {feed }}\right)^{0.5}[10]$. With $d_{\mathrm{s}}=500 \mu \mathrm{m}$, the 1D approximation of the heat flow induced by one isolated single pass of the laser beam is, therefore, justifiable for feed rates well above $0.03 \mathrm{~m} / \mathrm{s}$. When the ablation of a larger area is considered as sketched in Fig. 1, the relevant thermal diffusion length (assuming immediate reposition between the scan lines) is $\ell_{\text {diff }}=\left(4 \cdot \kappa \cdot\left(M \cdot d_{\ell} / v_{\text {feed }}\right)\right)^{0.5}$, where $M=160$ is the number of processing lines considered in this study. This diffusion length is found to be smaller than the width $b=10 \mathrm{~mm}$ of the processed square as long as the feed rate exceeds $0.24 \mathrm{~m} / \mathrm{s}$. The lowest applied feed rate in this study was $1 \mathrm{~m} / \mathrm{s}$, and therefore, we may assume 1D heat flow for all applied parameters.

When ablating several consecutive layers, one should also take into account that every processed layer may lead

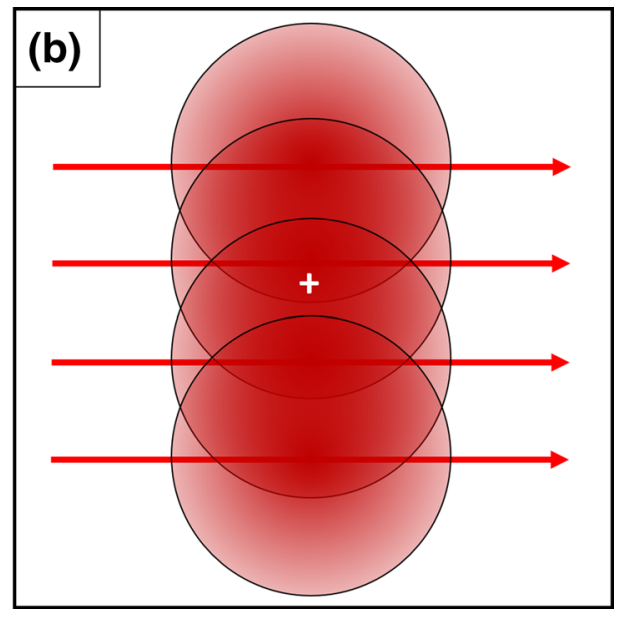

the left side resulting in a constant time interval between two successive passes. b Schematic illustration of the multiple interactions of the laser beam (indicated by the red circles) on a given point (white cross) which leads to heat accumulation between passes

to a change of the surface topography, which can lead to a change of the absorptance of the surface for one layer to the next [10-12].

\section{Analytical model of the heat accumulation}

The heat accumulation during pulsed laser materials processing has already been widely investigated $[1,2,7$, 10-12]. Every successive laser pulse contributes to the temperature increase of the workpiece. The absorbed part of the pulse energy $E_{\mathrm{P}}$ is determined by the absorptance $\eta_{\mathrm{abs}}$, which depends on the material (absorptivity) and the geometry of the interaction zone (multiple reflections). Part of the absorbed energy $\eta_{\mathrm{abs}} \cdot \mathrm{E}_{\mathrm{P}}$ is consumed for the actual ablation process but a fraction $\eta_{\text {heat }}$ of the absorbed energy remains in the workpiece as what is sometimes termed residual heat. The residual heat left in the workpiece after each laser pulse is, therefore, given by $Q=\eta_{\text {Heat }} \cdot \eta_{\mathrm{abs}} \cdot E_{\mathrm{P}}$. The material properties, the surface roughness, and the surface temperature influence both $\eta_{\text {abs }}$ and $\eta_{\text {Heat }}$ [13-16]. An average absorptance of $\eta_{\text {abs }}=0.55$ during processing of stainless steel AISI 316L was used for the modeling. For the residual heat we adopt $\eta_{\text {Heat }}=0.38$ as reported in [7].

\subsection{Heat accumulation between multiple pulses (HAP)}

The temperature distribution caused by the heat flow into the material depends on the deposited heat and on the material properties (density $\rho$, heat capacity $c_{\mathrm{p}}$, and thermal diffusivity $\kappa)$ of the processed workpiece. The basic equations describing 
the heat accumulation between consecutive heat inputs were derived in [1] together with a detailed discussion of the necessary assumptions. For 1D heat flow, the temporal evolution of the resulting temperature increase caused by HAP on the processed surface of the workpiece just before the incidence of the subsequent pulse can be approximated by [10]

$$
\begin{aligned}
\Delta T_{\mathrm{HAP}}(t)= & \frac{8 \cdot \eta_{\mathrm{abs}} \cdot \eta_{\text {heat }} \cdot E_{\mathrm{P}} \cdot \sqrt{f}}{\pi \cdot d_{\mathrm{s}}^{2}} \cdot \frac{1}{\rho \cdot c_{\mathrm{p}} \cdot \sqrt{4 \cdot \pi \cdot \kappa}} \\
& \cdot(2 \sqrt{f \cdot t}-1.46),
\end{aligned}
$$

where $f$ is the pulse repetition rate, $d_{\mathrm{s}}$ is the diameter of the laser beam on the surface of the workpiece, and $t>0$ is the duration of the processing. The first term is the (residual) heat input, the second term is given by the material properties, and the last term determines the temporal evolution of the temperature increase. The dashed green line in Fig. 2 shows the calculated temperature increase occurring during processing of AISI $316 \mathrm{~L}$ (material properties at $20{ }^{\circ} \mathrm{C}$ : $\rho=8000 \mathrm{~kg} / \mathrm{m}^{3}, c_{\mathrm{p}}=500 \mathrm{~J} /(\mathrm{kg} \mathrm{K}), \kappa=3.75 \times 10^{-6} \mathrm{~m}^{2} / \mathrm{s}$, $\eta_{\text {abs }}=0.55, \eta_{\text {Heat }}=0.38$ [17]) with a resting laser beam with $E_{\mathrm{P}}=175 \mu \mathrm{J}$ and $f=300 \mathrm{kHz}, d_{\mathrm{s}}=90 \mu \mathrm{m}$. The red line represents the melting temperature when assuming an initial temperature of $22{ }^{\circ} \mathrm{C}$. When the considered laser beam is moved over the workpiece with a velocity of $v_{\text {feed }}=2.0 \mathrm{~m} / \mathrm{s}$,

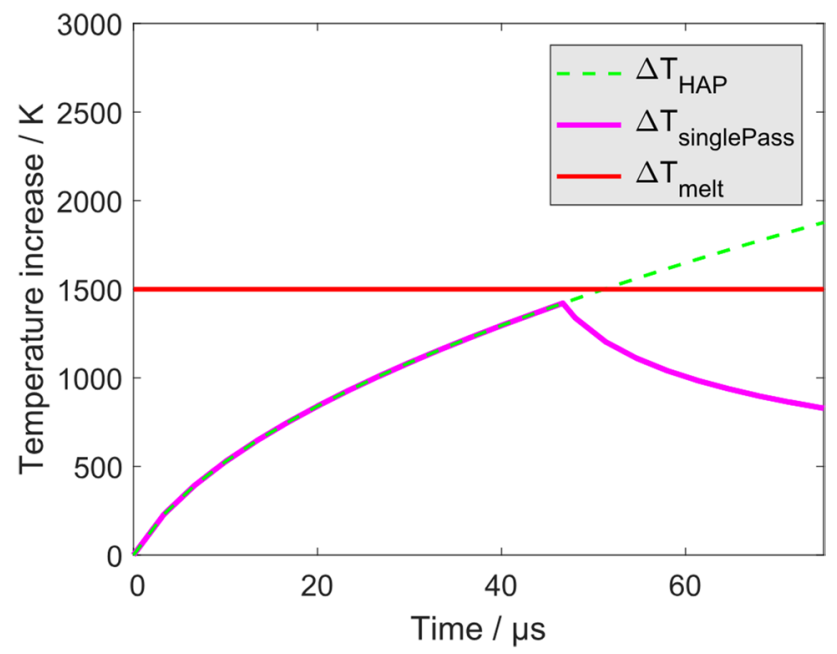

Fig. 2 Example of the temperature increase caused by HAP at a given point on the surface of a CrNi-Steel sample as a function of time. The green-dashed line illustrates the temperature increase caused by uninterrupted processing as given by Eq. (1). The magenta curve illustrates the temperature evolution occurring when the irradiation at the considered point is limited in time due to the moving beam as given by Eq. (2). The red line represents the temperature increase at which the melting temperature is reached assuming an initial surface temperature of $22{ }^{\circ} \mathrm{C}$. Parameters: $\eta_{\text {abs }}=0.55, \eta_{\text {Heat }}=0.38, \rho=8000 \mathrm{~kg} /$ $\mathrm{m}^{3}, c_{\mathrm{p}}=500 \mathrm{~J} /(\mathrm{kg} \mathrm{K}), \kappa=3.75 \times 10^{-6} \mathrm{~m}^{2} / \mathrm{s}, E_{\mathrm{P}}=175 \mu \mathrm{J}, f=300 \mathrm{kHz}$, $d_{\mathrm{s}}=90 \mu \mathrm{m}, v_{\text {feed }}=2.0 \mathrm{~m} / \mathrm{s}$ the irradiation at a given point on the surface ends after the time $t_{\text {irr }}=d_{\mathrm{s}} / v_{\text {feed }}=45 \mu \mathrm{s}$.

To model the decreasing temperature after the end of the irradiation time, according to [18] Eq. (1) can be extended by adding an identical negative heat source for the times $t>t_{\text {irr }}=d_{\mathrm{s}} / v_{\text {feed }}$. The modified Eq. (1) then reads

$\Delta T_{\text {singlePass }}(t)=\Delta T_{\mathrm{HAP}}(t)-\Theta\left(t-t_{\text {irr }}\right) \cdot \Delta T_{\mathrm{HAP}}\left(t-t_{\text {irr }}\right)$,

where $\Theta$ is the Heaviside step function. For the above example, the solution of Eq. (2) is shown by the magenta line in Fig. 2. For $t<t_{\text {irr }}$ Eq. (2) is identical to Eq. (1). For $t \geq t_{\text {irr }}$ the considered point on the surface is not heated anymore and the cooling caused by the heat dissipation into the workpiece leads to the gradually diminishing temperature as illustrated by the magenta line in Fig. 2.

\subsection{Heat accumulation between multiple passes (HAS-I)}

Based on Eq. (2), one can easily model the temperature evolution occurring during a process with multiple passes of the laser beam over one and the same point (HAS-I) by

$\Delta T_{\text {HAS-I }}(t)=\sum_{N=1}^{N_{\text {passes }}} \Delta T_{\text {singlePass }}\left(t-(N-1) \cdot t_{\text {pass }}\right)$,

where $t_{\text {pass }}$ is the time elapsing between two consecutive passes of the laser beam over the same spot, and $N \in \mathbb{N}$.

With the process strategy sketched in Fig. 1a, the time between successive passes over a given point is given by $t_{\text {pass }}$ $=\ell / v_{\text {feed }}+\left(\ell^{2}+d_{\ell}^{2}\right)^{0.5 / v_{\text {pos }}}$, where $v_{\text {pos }}$ is the feed during repositioning. When $v_{\text {feed }} \ll v_{\text {pos }}$, the second term can be neglected.

\subsection{Heat accumulation between multiple layers (HAS-II)}

For the sake of simplicity, we assume that every layer is processed with the same scanning strategy, hence without rotating the scanning pattern, with the same feed of the laser beam on the surface, and with the same process parameters. When $t_{\text {pass }}$ and the number of lines $M$ is known, the time interval between two consecutive layers is $t_{\text {layer }}=M \cdot t_{\text {pass }}$. For the scanning strategy shown in Fig. 1a, the temperature increase caused during the ablation of the surface layer by layer yields

$$
\begin{aligned}
\Delta T_{\text {HAS-II }}(t)= & \sum_{K=1}^{K_{\text {layers }}} \sum_{N=1}^{N_{\text {passes }}} \Delta T_{\text {singlePass, } 1 \mathrm{D}}\left(t-\left[(K-1) \cdot t_{\text {layer }}(4)\right.\right. \\
& \left.\left.+(N-1) \cdot t_{\text {pass }}\right]\right)
\end{aligned}
$$

where $K \in \mathbb{N}$, and $N \in \mathbb{N}$. 


\section{Surface structuring of AISI 316L}

When the accumulated heat leads to a temperature of at least $T_{\text {bump }}=607^{\circ} \mathrm{C}$, the ablated surface of the CrNi-Steel AISI 304 was found to be covered with small bumps with sizes in the range of few microns [7]. We have experimentally investigated this transition using rolled, non-treated square plates $\left(50 \times 50 \mathrm{~mm}^{2}\right)$ of AISI $316 \mathrm{~L}\left(\rho=8000 \mathrm{~kg} / \mathrm{m}^{3}\right.$, $\left.c_{\mathrm{p}}=500 \mathrm{~J} /(\mathrm{kg} \mathrm{K}), \kappa=3.75 \times 10^{-6} \mathrm{~m}^{2} / \mathrm{s}, \eta_{\text {heat }}=0.38\right)$ having a thickness of $2 \mathrm{~mm}$. The workpiece was mounted on a flat steel block $\left(70 \times 100 \times 10 \mathrm{~mm}^{3}\right)$ which served as a heat sink. The laser-processed surfaces were examined by means of SEM and a white-light interferometer. The experiments were performed with a home-build mJ-ps-laser operating at a wavelength of $\lambda=1030 \mathrm{~nm}$, with a pulse repetition rate of $f=300 \mathrm{kHz}$, and a pulse duration of $\tau=8 \mathrm{ps}$. The workpiece was placed in the defocus of the focusing optics in a way to result in a beam diameter on the surface of the workpiece of $d_{\mathrm{s}}=500 \mu \mathrm{m}$ resulting in a mean fluence of $H=0.71 \mathrm{~J} /$ $\mathrm{cm}^{2} \pm 0.06 \mathrm{~J} / \mathrm{cm}^{2}$. The uncertainty of the fluence results mainly from the experimental determination of the beam diameter of about $\pm 4 \%$. The scanning pattern consisted of 160 parallel arranged lines (see Fig. 1a) with a length of $\ell=10 \mathrm{~mm}$. The hatching distance $d_{\ell}$ was either 62.5 or $125 \mu \mathrm{m}$ resulting in $N_{\text {passes }}=8$ or $N_{\text {passes }}=4$, respectively. Since the experiments took place at room temperature, the temperature increase required to reach $T_{\text {bump }}$ was given by $\Delta T_{\text {bump }}=585 \mathrm{~K}$ and the temperature increase at which $T_{\text {melt }}$ was exceeded was $\Delta T_{\text {melt }}=1500 \mathrm{~K}$. Which of the two temperatures constitutes a process limit depends on the application and the desired processing result. A mean fluence of $H=0.71 \mathrm{~J} / \mathrm{cm}^{2}$ was used together with different feed rates to investigate the relevance of $T_{\text {bump }}$ as well as $T_{\text {melt }}$. The applied mean fluence is well above the ablation threshold $0.1 \mathrm{~J} / \mathrm{cm}^{2}$ of stainless steel [19-21]. The feed rates were adapted in a way to result in maximum surface temperatures well below as well as above the temperatures $T_{\text {bump }}$ and $T_{\text {melt }}$ during processing a single layer. The transition over the critical temperature $T_{\text {bump }}$ was additionally investigated to analyze the influence of the changing absorptance during multiple-layer processing [22].

\subsection{Single-layer process}

\subsubsection{Calculations}

The temperature increase due to heat accumulation (HAP and HAS-I) was calculated with Eq. (3) using the material parameters listed above. Four different feed rates were applied for a mean fluence of $0.71 \mathrm{~J} / \mathrm{cm}^{2}: 1.0 \mathrm{~m} / \mathrm{s}, 5.0 \mathrm{~m} / \mathrm{s}$, $10.0 \mathrm{~m} / \mathrm{s}$, and $20.0 \mathrm{~m} / \mathrm{s}$. The uncertainty of every applied feed rate is due to the accuracy of the galvo-mirrors in

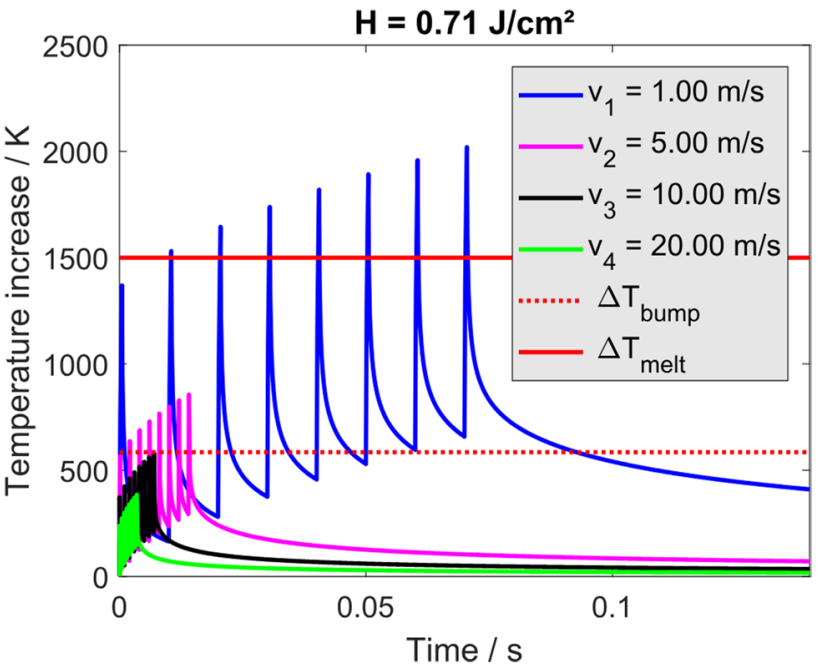

Fig. 3 Calculated temperature increase caused by HAP and HAS-I as given by Eq. (3) for a point on the surface of polished $\left(S_{\mathrm{a}}=0.2 \mu \mathrm{m}\right)$ AISI $316 \mathrm{~L}$ as a function of time processed with a mean fluence of $0.71 \mathrm{~J} / \mathrm{cm}^{2}$ per pulse and different feed rates. Further parameters: $\eta_{\mathrm{abs}}=0.55, \eta_{\text {Heat }}=0.38, \rho=8000 \mathrm{~kg} / \mathrm{m}^{3}, c_{\mathrm{p}}=500 \mathrm{~J} /(\mathrm{kg} \mathrm{K})$, $\kappa=3.75 \times 10^{-6} \mathrm{~m}^{2} / \mathrm{s}, f=300 \mathrm{kHz}, d_{\mathrm{s}}=500 \mu \mathrm{m}, N_{\text {passes }}=8$

the scanner and was about $2 \%$. Figure 3 shows the temperature increase expected for the said processes. Since $N_{\text {passes }}=8$ (see above) the temperature reaches eight peaks. The temporal distance between the eight peaks, and therefore, the duration of the cooling phase between the upcoming and the current beam pass decreases with increasing feed rate. Due to the heat accumulation the upcoming peak is higher than the current one. For the considered mean fluence of $0.71 \mathrm{~J} / \mathrm{cm}^{2}$ a feed of $20.0 \mathrm{~m} / \mathrm{s}$ (green line in Fig. 3) results in a maximum temperature increase of $\Delta T=385 \mathrm{~K}$ which is well below $\Delta T_{\text {bump }}$. The surface resulting from this process should be smooth. Decreasing the feed rate to $10 \mathrm{~m} / \mathrm{s}$ (black line in Fig. 3) leads to a maximum temperature increase of $\Delta T=585 \mathrm{~K}$ which is slightly below $\Delta T_{\text {bump }}$. The surface resulting from this process may therefore show the transition from a smooth surface to a bumpy surface. Further decreasing of the feed rate to $5.0 \mathrm{~m} / \mathrm{s}$ (magenta line in Fig. 3) leads to a maximum temperature increase of $\Delta T=860 \mathrm{~K}$ which is well below $\Delta T_{\text {melt }}$ and significantly above $\Delta T_{\text {bump }}$. The surface resulting from this process should be covered with well-pronounced grooves. Applying a feed rate of $1.0 \mathrm{~m} / \mathrm{s}$ (blue line in Fig. 3) results in a maximum temperature increase of $\Delta T=2030 \mathrm{~K}$ which is well above $\Delta T_{\text {melt }}$. The surface resulting after this process is expected to show signs of solidified melt, and therefore, significantly differ compared to a surface processed with the higher feed rates. 


\subsubsection{Experiments}

The above predictions on the expected surface quality were verified by experiments with the same parameters as used to calculate the results shown in Fig. 3. Figure 4 shows SEM images of the surfaces that were processed with a mean fluence of $0.71 \mathrm{~J} / \mathrm{cm}^{2}$ per pulse and the feed rates $1.0 \mathrm{~m} / \mathrm{s}$, $5.0 \mathrm{~m} / \mathrm{s}, 10.0 \mathrm{~m} / \mathrm{s}$, and $20.0 \mathrm{~m} / \mathrm{s}$. As expected, the processed areas show laser-induced periodic surface structures (LIPSS). According to [23], the LIPSS can be classified in ripples, grooves, and spikes, where grooves and spikes correspond to a bumpy surface structure [7]. For the highest feed rate of $20.0 \mathrm{~m} / \mathrm{s}$, only ripples can be recognized in Fig. 4a. As expected from theory, the critical temperature required for the formation of bumps (grooves and spikes) was not reached. Decreasing the feed rate to $10.0 \mathrm{~m} / \mathrm{s}$ leads to the creation of fine grooves and ripples on the surface (see Fig. 4b) as the temperature according to theory is in the range of $T_{\text {bump. }}$. Applying a feed rate of $5.0 \mathrm{~m} / \mathrm{s}$ leads to a surface that exhibits grooves covered with ripples as shown by Fig. 4c. The occurrence of grooves confirms the theoretical expectation that at these parameters the temperature increase exceeds $\Delta T_{\text {bump }}$ which according to [7] leads to a bumpy surface. Further decreasing the feed to $1.0 \mathrm{~m} / \mathrm{s}$ leads to a surface which is covered with micro-holes (Fig. 4d) which is a clear sign that the laser pulses interacted with a liquid surface. Hence, the experimental results are well consistent with the above theory.
To quantify the process-resulting roughness, all surfaces shown in Fig. 4 were additionally examined by means of a white-light interferometer to determine the mean roughness depth $S_{\mathrm{Rz}}$. We performed five measurements on every processed area as well as on an untreated area. In Table 1 the mean values of $S_{\mathrm{Rz}}$ with corresponding standard deviation are listed for all resulting surface structures shown in Fig. 4. The mean roughness depth of an untreated area is $0.076 \mu \mathrm{m}$. After processing the mean roughness depth is significantly increased, e.g., applying a feed rate of $20 \mathrm{~m} / \mathrm{s}$ results in a mean roughness depth of $1.298 \mu \mathrm{m}$. When the feed rate is decreased the mean roughness depth increases further. This is tantamount to rougher surfaces (see Fig. 4), and therefore, the mean roughness depth is a suitable parameter to describe the transitions from a smooth surface (covered only with ripples) to a bumpy surface.

Table 1 Measured mean roughness depth $S_{\mathrm{Rz}}$ of an untreated surface and the structured surfaces dependent on the applied feed rate. Further parameters: $\lambda=1030 \mathrm{~nm}, f=300 \mathrm{kHz}, d_{\mathrm{s}}=500 \mu \mathrm{m}, H=0.71 \mathrm{~J} /$ $\mathrm{cm}^{2}, d_{\ell}=62.5 \mu \mathrm{m}, N_{\text {passes }}=8$

\begin{tabular}{lc}
\hline$v / \mathrm{m} / \mathrm{s}$ & $\begin{array}{l}\text { Mean roughness } \\
\text { depth } S_{\mathrm{Rz}} / \mu \mathrm{m}\end{array}$ \\
\hline Untreated surface & $0.076 \pm 0.004$ \\
20.0 & $1.298 \pm 0.039$ \\
10.0 & $2.088 \pm 0.035$ \\
5.0 & $4.509 \pm 0.857$ \\
1.0 & $6.443 \pm 0.950$ \\
\hline
\end{tabular}

Fig. 4 LIPSS on polished $\left(S_{\mathrm{a}}=0.2 \mu \mathrm{m}\right)$ AISI $316 \mathrm{~L}$ formed by processing with a mean fluence of $H=0.71 \mathrm{~J} / \mathrm{cm}^{2}$ per pulse and the different feed rates of $20.0 \mathrm{~m} / \mathrm{s}(\mathbf{a}), 10.0 \mathrm{~m} / \mathrm{s}(\mathbf{b})$, $5.0 \mathrm{~m} / \mathrm{s}(\mathbf{c})$, and $1.0 \mathrm{~m} / \mathrm{s}(\mathbf{d})$. The red arrow indicates the direction of the feed for all applied feed rates. Further parameters: $\lambda=1030 \mathrm{~nm}, f=300 \mathrm{kHz}$, $d_{\mathrm{s}}=500 \mu \mathrm{m}, d_{\ell}=62.5 \mu \mathrm{m}$, $N_{\text {passes }}=8$
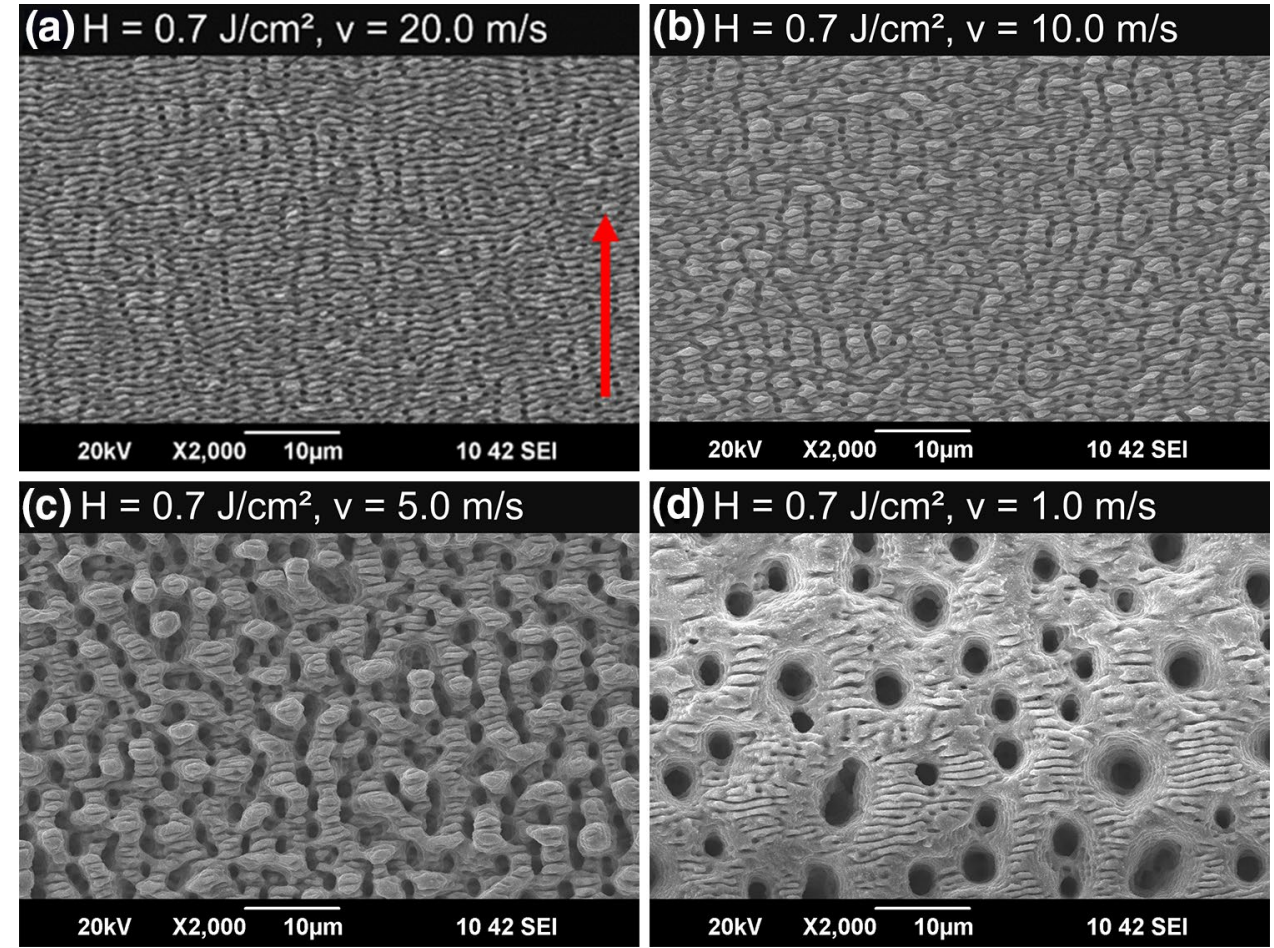


\subsection{Multi-layer process}

\subsubsection{Calculations}

For the investigation of the additional impact of HAS-II we consider a mean fluence of $0.71 \mathrm{~J} / \mathrm{cm}^{2}$ combined with a feed rate of $10.0 \mathrm{~m} / \mathrm{s}$ and a hatching distance of $d_{\ell}=125 \mu \mathrm{m}$ resulting in $N_{\text {passes }}=4$ to reduce the maximum temperature increase per layer. According to Fig. 3, this leads to maximum temperature increase of $490 \mathrm{~K}$ during the first layer which is just $110 \mathrm{~K}$ below $\Delta T_{\text {bump }}$. The complete temperature increase caused by all three heat accumulation effects together (HAP, HAS-I, and HAS-II) was calculated with Eq. (4), again with the same material and scanning parameters as listed above. All material parameters were assumed to be constant. Taking this into account, the temperature increase calculated with Eq. (4) for the process of the first 50 layers is shown in Fig. 5. According to the used parameters $N_{\text {passes }}$ equals 4 (compare Fig. 3). Hence, the peaks seen in Fig. 5 actually are four peaks that, however, are not resolved in the picture as they are so close to each other. One can see that the critical temperature increase $\Delta T_{\text {bump }}$ is reached in the range around the eighth and tenth layer.

\subsubsection{Experiments}

The state of the surfaces that were observed after the ablation of different numbers of layers using the same parameters as discussed above are shown by the SEM images

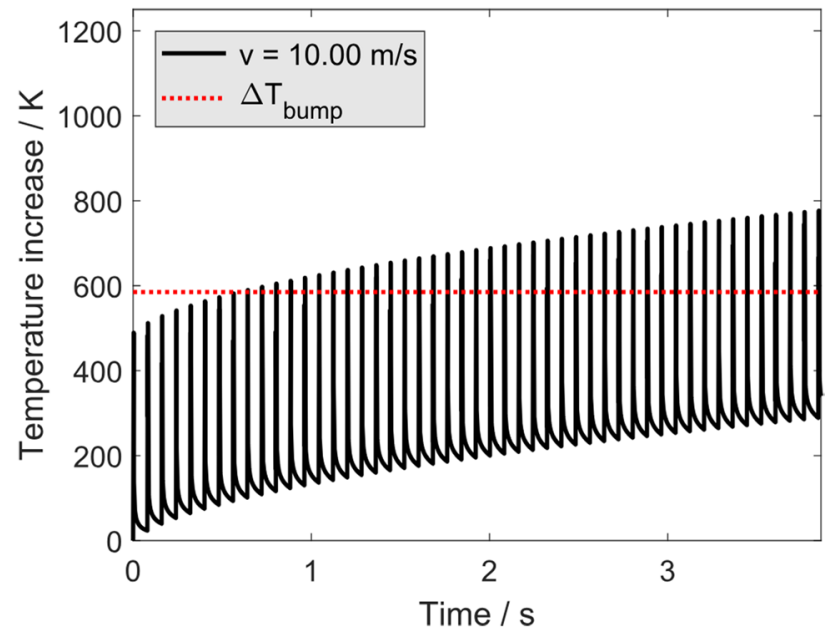

Fig. 5 Calculated temperature increase caused by the combination of HAP, HAS-I, and HAS-II (Eq. 4) at a given point on a polished $\left(S_{\mathrm{a}}=0.2 \mu \mathrm{m}\right)$ AISI 316L surface on which 50 layers where processed using the scanning pattern specified in Fig. 1. Parameters: $\eta_{\mathrm{abs}}=0.55$, $\eta_{\text {Heat }}=0.38, \rho=8000 \mathrm{~kg} / \mathrm{m}^{3}, c_{\mathrm{p}}=500 \mathrm{~J} /(\mathrm{kg} \mathrm{K}), \kappa=3.75 \times 10^{-6} \mathrm{~m}^{2} / \mathrm{s}$, $\lambda=1030 \mathrm{~nm}, f=300 \mathrm{kHz}, E_{\mathrm{P}}=1.4 \mathrm{~mJ}, d_{\mathrm{s}}=500 \mu \mathrm{m}, H=0.71 \mathrm{~J} / \mathrm{cm}^{2}$, $v_{\text {feed }}=10.0 \mathrm{~m} / \mathrm{s}, N_{\text {passes }}=4$ in Fig. 6. After the ablation of the first layer (Fig. 6a), the resulting surface is homogeneously covered with fine ripples. Processing two layers (Fig. 6b) leads to the formation of more pronounced ripples. This still applies for the resulting surface after the process of five layers as shown by Fig. 6c. Nevertheless, rougher ripples can be recognized after processing five layers. The surface may start to be in transition from a smooth surface to a bumpy surface. After the process of the tenth layer (Fig. 6d) the formation of fine micro-grooves perpendicular to the ripples can be recognized. The surface is in transition from a smooth surface to a bumpy surface. This is consistent with the theoretical prediction that the transition to a bumpy surface should occur after processing eight to ten layers, see Fig. 5. This surface appearance is maintained also after the ablation of further layers, as shown by the image taken after the ablation of 20 layers given in Fig. 6e. The grooves have a periodicity of a few microns. When the number of processed layers is further increased to 50 (Fig. 6f) more pronounced grooves with a higher periodicity are formed.

Again, all surfaces shown in Fig. 6 were examined by means of a white-light interferometer to determine the mean roughness depth $S_{\mathrm{Rz}}$. The results are listed in Table 2. The more layers were processed the larger is the resulting mean roughness depth. This is tantamount to rougher surfaces (see Fig. 6), and therefore, the mean roughness depth is a suitable parameter to describe the transitions from a smooth surface (covered only with ripples) to a bumpy surface.

\section{Conclusion}

The known fundamental equation describing the temperature increase caused by heat accumulation between consecutive heat inputs and 1D heat flow into the workpiece was used to develop a simple analytical model to calculate the temperature increase that occurs during materials processing with multiple parallel scanning passes (HAS-I) and the ablation of multiple layers (HAS-II). The model was shown to be consistent with the experimental findings by analyzing the resulting surface topography produced by laser processing. Modeling of situations with 2D or 3D heat flow would follow the same approach as outlined here just using the known corresponding $2 \mathrm{D}$ and $3 \mathrm{D}$ versions of the fundamental heat accumulation equations.

The explicit expressions depend on the applied scanning pattern, as illustrated by the additional example in the "Appendix". 
Fig. 6 LIPSS on polished $\left(S_{\mathrm{a}}=0.2 \mu \mathrm{m}\right)$ AISI 316L formed by laser processing of $1(\mathbf{a}), 2$ (b), 5 (c), $10(\mathbf{d}), 20(\mathbf{e})$, and 50 (f) layers using a mean fluence of $H=0.71 \mathrm{~J} / \mathrm{cm}^{2}$ per pulse and a feed rate of $v_{\text {feed }}=10.0 \mathrm{~m} / \mathrm{s}$. The red arrow indicates the direction of the feed for all applied feed rates. Further parameters: $\lambda=1030 \mathrm{~nm}$, $f=300 \mathrm{kHz}, E_{\mathrm{P}}=1.4 \mathrm{~mJ}$, $d_{\mathrm{s}}=500 \mu \mathrm{m}, N_{\text {passes }}=4$

Table 2 Measured mean roughness depth $S_{\mathrm{Rz}}$ of an untreated surface and the structured surfaces dependent on the number of processed layers. Further parameters: $\lambda=1030 \mathrm{~nm}, f=300 \mathrm{kHz}$, $d_{\mathrm{s}}=500 \mu \mathrm{m}, H=0.71 \mathrm{~J} /$ $\mathrm{cm}^{2}, \nu=10 \mathrm{~m} / \mathrm{s}, d_{\ell}=125 \mu \mathrm{m}$, $N_{\text {passes }}=4$
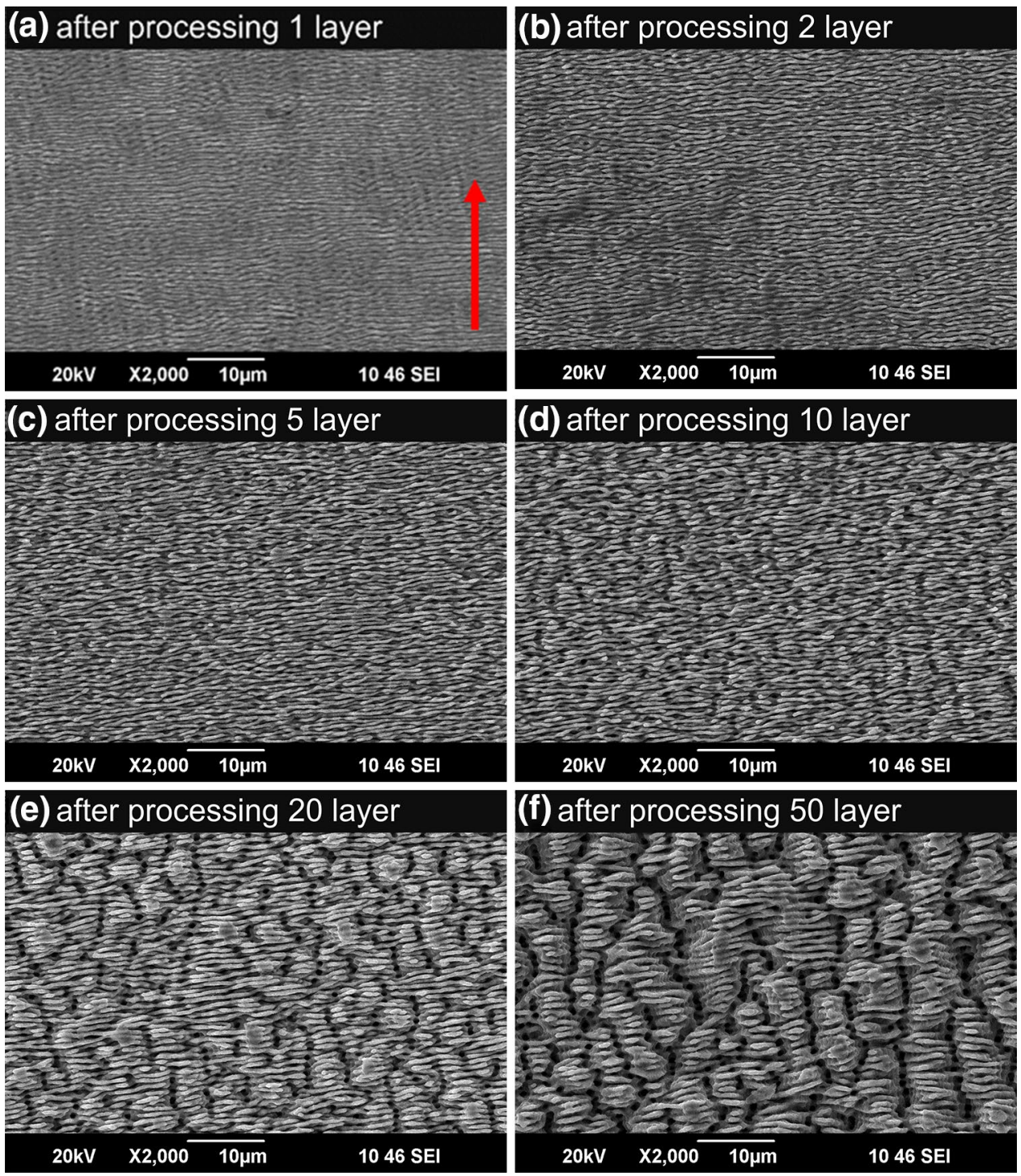

Acknowledgements This project has received funding from the European Union's Horizon 2020 Research and Innovation Programme under Grant Agreement No. 687613.

\begin{tabular}{ll}
\hline $\begin{array}{l}\text { Number of } \\
\text { processed } \\
\text { layers }\end{array}$ & $\begin{array}{l}\text { Mean roughness } \\
\text { depth } S_{\mathrm{Rz}} / \mu \mathrm{m}\end{array}$ \\
\hline $\begin{array}{l}\text { Untreated } \\
\text { surface }\end{array}$ & $0.076 \pm 0.004$ \\
1 & $0.972 \pm 0.039$ \\
2 & $1.162 \pm 0.035$ \\
5 & $1.574 \pm 0.030$ \\
10 & $2.040 \pm 0.055$ \\
20 & $2.797 \pm 0.142$ \\
50 & $4.720 \pm 0.098$ \\
\hline
\end{tabular}

Open Access This article is distributed under the terms of the Creative Commons Attribution 4.0 International License (http://creativeco mmons.org/licenses/by/4.0/), which permits unrestricted use, distribution, and reproduction in any medium, provided you give appropriate credit to the original author(s) and the source, provide a link to the Creative Commons license, and indicate if changes were made.

\section{Appendix}

The scanning strategy shown in Fig. 7a is characterized by alternating feed direction along the adjacent parallel paths and results in shorter positioning ways between two successive lines, and therefore, leads to a shorter total processing time as the example discussed above. When the considered 


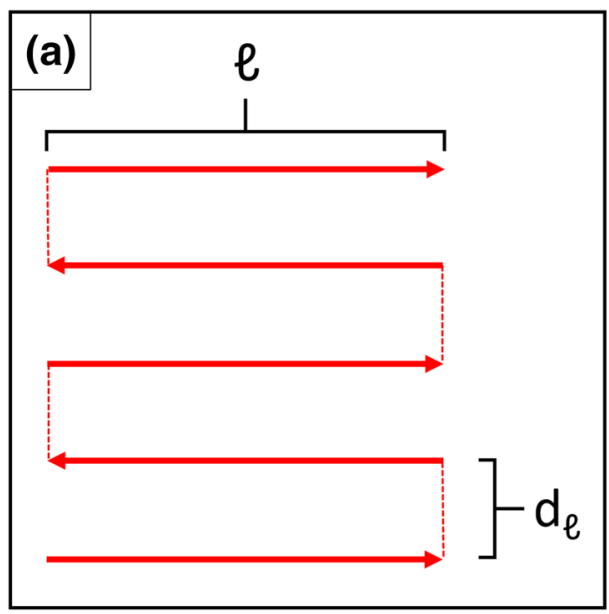

Fig. 7 a Schematic illustration of an alternative scanning strategy. The solid red arrows show the movement of the laser beam during processing, the dashed lines indicate the repositioning of the beam (during which the laser is off). $\ell$ is the length of the processing paths and $d_{\ell}$ the distance between adjacent paths. The direction of the feed alternates resulting in an alternating time interval between two successive passes.

point is located at the center of the processing lines, the time interval between the successive passes of the laser beam at this point is constant for the reason of symmetry. When leaving the center of the processing lines two different time intervals between two successive passes occur due to the asymmetric beam trajectory as indicated by Fig. $7 \mathrm{~b}$.

For parallel arranged lines with alternating feed direction, there can be two different processing times of a single pass $\left(t_{\text {short }}\right.$ and $\left.t_{\text {long }}\right)$ depending on the current processing line. Since both processing times of a single pass are repetitive, the chronical interval depends on the parity of the processing line. Defining rightwards passes where the laser beam moves from the left side to the right side as lines with even parity leads to the temperature increase $\Delta T_{\text {HAS-I }}$ and can be expressed by

$\Delta T_{\text {HAS-II }}(t)=\sum_{K=1}^{K_{\text {layers }}} 1 \cdot\left\{\begin{array}{l}\sum_{N=1}^{N_{\text {passes }}} \Delta T_{\text {singlePass, } 1 \mathrm{D}}\left(t-\left[(K-1) \cdot t_{\text {layer }}+(N-1) \cdot t_{\text {short }}\right]\right), N=2 k, \\ \sum_{N=1}^{N} \Delta T_{\text {singlePass, } 1 \mathrm{D}}\left(t-\left[(K-1) \cdot t_{\text {layer }}+(N-1) \cdot t_{\text {long }}\right]\right), N=2 k+1,\end{array}\right.$

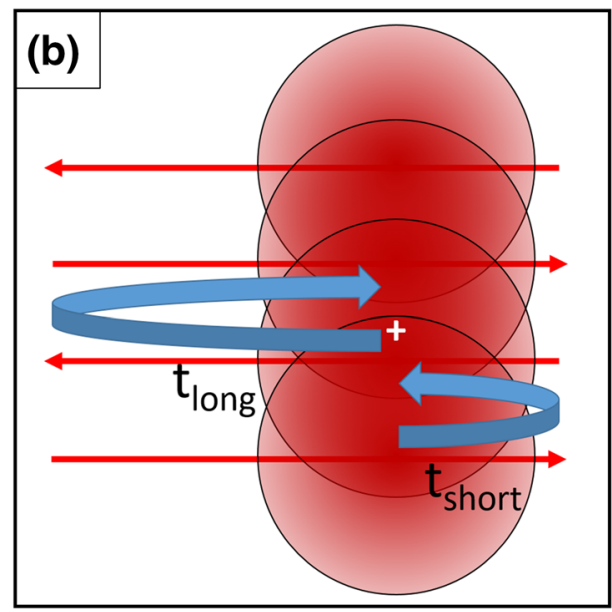

b schematic illustration of the multiple interactions of the laser beam (indicated by the red circles) on a given point out of center (white cross) which leads to heat accumulation between passes with alternating time intervals $t_{\text {short }}$ and $t_{\text {long }}$ between two successive passes (blue arrows)

$\Delta T_{\text {HAS-I }}(t)=\left\{\begin{array}{l}\sum_{N=1}^{N_{\text {passes }}} \Delta T_{\text {singlePass }}\left(t-(N-1) \cdot t_{\text {short }}\right), \quad N=2 k, \\ \sum_{N=1}^{N} \Delta T_{\text {singlePass }}\left(t-(N-1) \cdot t_{\text {long }}\right), N=2 k+1,\end{array}\right.$

where $N \in \mathbb{N}$, and $k \in \mathbb{N}$.

For the sake of simplicity, we assume that every layer is processed with the same scanning strategy, hence without rotating the scanning pattern, with the same feed of the laser beam on the surface, and with the same process parameters. When $t_{\text {short }}, t_{\text {long }}$, and the number of lines $M$ is known the time interval between two consecutive layers is $t_{\text {layer }}=M \cdot\left(t_{\text {short }}+t_{\text {long }}\right) / 2$. For the scanning strategy shown in Fig. 7 a the temperature increase caused during the ablation of the surface layer by laser yields 
where $K \in \mathbb{N}, N \in \mathbb{N}$, and $k \in \mathbb{N}$.

The introduced model can be used to calculate the temperature increase due to heat accumulation for all scanning strategies that can be parameterized.

\section{References}

1. R. Weber, T. Graf, P. Berger, V. Onuseit, M. Wiedenmann, C. Freitag, A. Feuer, Opt. Express 22, 11312 (2014)

2. T.V. Kononenko, C. Freitag, M.S. Komlenok, V. Onuseit, R. Weber, T. Graf, V.I. Konov, J. Appl. Phys. 118, 103105 (2015)

3. R. Weber, C. Freitag, T.V. Kononenko, M. Hafner, V. Onuseit, P. Berger, T. Graf, Phys. Procedia 39, 137 (2012)

4. B. Wu, M. Zhou, J. Li, X. Ye, G. Li, L. Cai, Appl. Surf. Sci. 256, 61 (2009)

5. P. Bizi-Bandoki, S. Benayoun, S. Valette, B. Beaugiraud, E. Audouard, Appl. Surf. Sci. 257, 5213 (2011)

6. N.A. Patankar, Langmuir ACS J. Surf. Colloids 20, 8209 (2004)

7. F. Bauer, A. Michalowski, T. Kiedrowski, S. Nolte, Opt. Express 23, 1035 (2015)

8. J.-P. Negel, A. Voss, M. Abdou Ahmed, D. Bauer, D. Sutter, A. Killi, T. Graf, Opt. Lett. 38, 5442 (2013)

9. H. Hügel, T. Graf, Laser in der Fertigung: Strahlquellen, Systeme, Fertigungsverfahren: Strahlquellen, Systeme, Fertigungsverfahren (Springer Fachmedien Wiesbaden GmbH, Wiesbaden, 2014)
10. R. Weber, T. Graf, C. Freitag, A. Feuer, T. Kononenko, V.I. Konov, Opt. Express 25, 3966 (2017)

11. B.N. Chichkov, C. Momma, S. Nolte, F. Alvensleben, A. Tünnermann, Appl. Phys. A 63, 109 (1996)

12. E.G. Gamaly, A.V. Rode, B. Luther-Davies, V.T. Tikhonchuk, Phys. Plasmas 9, 949 (2002)

13. D. Bergström, J. Powell, A.F.H. Kaplan, Appl. Surf. Sci. 253, 5017 (2007)

14. H. Kwon, W.-K. Baek, M.-S. Kim, W.-S. Shin, J.J. Yoh, Opt. Lasers Eng. 50, 114 (2012)

15. A.Y. Vorobyev, C. Guo, Appl. Phys. Lett. 86, 11916 (2005)

16. A.Y. Vorobyev, C. Guo, Opt. Express 14, 13113 (2006)

17. Deutsche Edelstahlwerke GmbH, Werkstoffdatenblatt 1.4404 / AISI 316L, https://www.dew-stahl.com/fileadmin/files/dewstahl.com/documents/Publikationen/Werkstoffdatenblaetter/ RSH/1.4404_de.pdf (2016). Accessed 2017

18. H.S. Carslaw, J.C. Jaeger, Conduction of heat in solids (Clarendo Press, Oxford, 1967)

19. S. Döring, S. Richter, S. Nolte, A. Tünnermann, Opt. Express 18, 20395 (2010)

20. B. Jaeggi, B. Neuenschwander, M. Schmid, M. Muralt, J. Zuercher, U. Hunziker, Phys. Procedia 12, 164 (2011)

21. G. Raciukaitis, M. Brikas, P. Gecys, M. Gedvilas, Proc. SPIE 7005, 70052L (2018)

22. A.Y. Vorobyev, C. Guo, Appl. Phys. A 86, 321 (2007)

23. G.D. Tsibidis, C. Fotakis, E. Stratakis, Phys. Rev. B 92, 041405 (2015) 